\title{
Inventory Management using Matrix Analysis and Inventory Index in an Oncology Pharmacy of a Tertiary Care Teaching Hospital
}

\author{
Subratha C' ${ }^{1}$ Naveen Kumar ${ }^{2}$, Bryal D' Souza ${ }^{3 *}$, Arun Mavaji ${ }^{4}$, Rajesh Kamath ${ }^{5}$ \\ 'Department of Public Health, MAHE, Manipal, Karnataka, INDIA. \\ ${ }^{2}$ Associate Professor, Department of Hospital Administration, KMC, MAHE, Manipal, Karnataka, INDIA. \\ ${ }^{3}$ Assistant Professor, PSPH, MAHE, Manipal, Karnataka, INDIA. \\ ${ }^{4}$ Hospital Administrator, MS Ramaiah Memorial Hospital, Bangalore, Karnataka, INDIA. \\ ${ }^{5}$ Assistant Professor, PSPH, MAHE, Manipal, Karnataka, INDIA.
}

\section{ABSTRACT}

Aim: To apply matrix analysis using the principles of Always Better Control(ABC), Vital, Essential and Desirable (VED) analysis and inventory index for inventory control and to identify the drugs that require management control and monitoring. Methods: Retrospective descriptive study using ABC, VED, Matrix analysis, inventory index and Perusal of records at the oncology pharmacy of a 2032 bedded multispecialty teaching hospital. Results: The study analyzed the 169 drugs in the oncology pharmacy for applying monitoring and control techniques. Only 29 out of the 169 oncology drugs were being consumed. $A B C$ analysis showed that of the 29 drugs under consumption, high value drugs ie category $A$ drugs were 4 in number(ie $3.1 \%$ ). Here, $10 \%$ of drugs accounted for $70 \%$ of monetary value. Category B contributed 4 drugs ie $(3.1 \%)$. Here, $20 \%$ of drugs accounted for $20 \%$ of monetary value. The remaining 21 drugs were under Category C. Here, $70 \%$ of drugs accounted for $10 \%$ of monetary value. The results of VED analysis showed one drug as Vital, 160 drugs as Essential and 8 drugs as Desirable. Matrix analysis aims at categorizing drugs based on vitality and cost. The matrix showed a total of 5 drugs in category $1: 4$ drugs being high cost essential dugs and one being a low cost vital drug;
\end{abstract}

156 drugs in Category II : consisting of 4 medium cost essential drugs and 152 low cost essential drugs. Cat III was constituted of 8 drugs that were low cost and desirable. The Inventory index was applied for category one drugs to check for stock turnover and availability. Conclusion: Matrix analysis using $A B C, V E D$ and inventory index can assist in the effective management of hospital inventory stocks. It provides management with actionable data on the areas of inventory that require control and supervisory measures.

Key words: $A B C$ analysis, matrix, inventory index, VED analysis.

Correspondence

Bryal dsouza, Assistant Professor, Department of Public Health, Manipal University, Karnataka, INDIA

Phone: 00919900405393

Email: brayal.dsouza@manipal.edu

DOI: 10.5530/jyp.2018.10.18

\section{INTRODUCTION}

Recent advancements in the system of medicine have evolved more complex, sophisticated, efficient, expensive line of treatment. in terms of medicine costs, equipment's and other factors. ${ }^{1}$ Among overall budget of hospital $1 / 3$ rd of which is used for purchasing and supply of medicines in the pharmacy. ${ }^{2,3,7}$

Management of pharmacy is a complex and critical process and is one of the areas in the hospital where large amount of money is invested on purchase on recurring basis with maximum return on investment. ${ }^{3}$ Pharmacy inventory management is a complex but critical process with in the health care delivery system. The affordability of care can have a direct effect the way pharmacies dispense and select the choices of drugs. Its main function is to record the drugs which enters newly or returned from the pharmacy. Utilizing drugs that are non-contracted or not in the formulary may be costlier to the patient or stringent regulatory requirements pertaining to drug traceability. ${ }^{4}$

The health managers are searching for alternative techniques to reduce the cost of inventories of pharmacy which lower the quality care of patients. A study suggested that review and control measures for Expensive drugs can bring about $20 \%$ savings in medical store budget. ${ }^{5,7}$

To yield maximize returns on investment at minimal costs, health care managers must adopt scientific methods. ${ }^{2,3,5}$ To achieve maximum output of the minimum inputs, stock analysis meets this by stretching the limited means to meet unlimited needs based on the economic principle. Each item in health care is uniquely critical and is perceived to supply high levels of service delivery ${ }^{5}$

$\mathrm{ABC}$ analysis is commonly known as 'Always Better Control' which is based on the Pareto's principal "separating vital few from the trivial many" which is popularly known as " $80 / 20$ rule" depending on capital investment of the drugs. ${ }^{3,6} \mathrm{ABC}$ analysis is based on monetary value and rate of consumption of the items. ${ }^{7}$ Leading plans on alternative stock arrangements, consignment stocks, reorder calculations can help to determine the inventory level for stricter monitoring (eg: A category drugs are monitored more frequently than $\mathrm{C}$ category drugs. ${ }^{8}$

This helps the inventory manager to attain and close monitor where greatest benefits in terms of cost reduction as well as maintain a continuous and uninterrupted movement of stock. This also helps in identification of drugs on which efforts can be concentrated profitably. It provides sound basis on which to allocate funds and time. It can be used to design cycle counting schemes (E.g: A items may be counted 3 times per year, B may be 1-2 times per year and C items may be once or not at all). ${ }^{8}$

$10-20 \%$ of items (A category) accounts for $70-80 \%$ of consumption, the next $15-25 \%$ (B category) accounts for $16-20 \%$ of the consumption and 
the balance $65-75 \%$ (C category) accounts for $5-10 \%$ of the consumption. (A category drugs are closely monitored because of the value involved $(70-80 \%))$

Some items which have low monetary value and lifesaving are not considered as important because they are not present in A category. ${ }^{2,3,6,8}$ To overcome the limitation of the ABC analysis VED analysis can be used which is based on criticality of the item. VED analysis also based on need of the drug in hospital and classified as 'Vital, Essential and Desirable'.

Where, 'V' (Vital) category drugs, without which hospital cannot perform its function i.e. patient care these category drugs, should have more safety stocks to ensure a higher degree of safety. Inventory manager should make sure that these drugs should be available for the patient all the times. 'E' (Essential) category drugs, without which hospital can function for few days but it may affect the quality of patient care. 'D' (Desirable) category drugs, without which may not affect the functioning of hospital for certain period of time. ${ }^{8}$

A combination of $\mathrm{ABC}$ and VED matrix analysis can be gainfully employed to evolve a meaningful control over the material supplied. ${ }^{7}$ In which Category I may be low cost one but critical for patient care and its more important ones. Category II drugs are of intermediate importance and Category III drugs are least importance and can be controlled by store keeper. ${ }^{8}$

Grouping will essentially depend upon the strategy of management and environment of functioning. These Simple techniques can be effective in materials management systems. Improving efficiency in material management is through improving the turnover of Inventory. ${ }^{9}$ The hospital under study tracked the consumption of inventory and evolved a new method called Inventory Index which helps in detecting overstock of drugs in pharmacy. ${ }^{9}$

In present study hospital the inventory was calculated by using formula, Sale/ procurement of drugs $=$ previous 3 -month average inventory ${ }^{\star} 26$ days - global stock

Stock $/$ month $=$ sales in last 7 days stock - current stock -7 days of last month stock

\section{MATERIAL AND METHODS}

The study was conducted at one of the largest multi-specialty tertiary care centers in south India. The data of annual consumption and expenditure incurred on each item of the medical store for the financial year 2015- 2016 were collected and then transcribed and analyzed in MS Excel. $\mathrm{ABC}$ analysis and VED analysis were done on the drug inventory of the oncology pharmacy to calculate and analyze the annual drug expenditure and criticality of drugs. Vitality of the drug were determined in consultation with the clinician and their consensus were taken. The matrix analysis was used to analyze the category of drugs requiring varying degrees of management control and supervision. Inventory index method was also used to forecast the stock turnover

\section{$\mathrm{ABC}$ analysis}

The list calculated from the available drugs worked out were firstly arranged in descending order, the cumulative percentage cost, cumulative frequency and cumulative percentage of number of items were calculated. The final list was then subdivided into three categories: A, B and C, based on the cumulative cost percentage of $70 \%, 20 \%$ and $10 \%$, respectively.

The annual expenditure of individual items was arranged in descending order. The cumulative cost of all the items was calculated. The cumulative percentage of expenditure and the cumulative percentage of number of items were calculated. This list was then subdivided into three categories:
A, B and C, based on the cumulative cost percentages of $70 \%, 20 \%$ and $10 \%$, respectively

\section{VED analysis}

Hospitals can't rely solely on $\mathrm{ABC}$ analysis which provides the annual consumption value, as some drugs may be of low consumption value but highly critical or lifesaving in patient care and survival. Therefore, arise the need for classifying drugs based on criticality.

The VED criticality analysis of all the listed items was performed by classifying the items into vital (V), essential (E) and desirable (D) categories. The items critically needed for the survival of the patients and those that must be available at all times, without the availability of which patient care can compromised and the non-availability of which hospital cannot function were included in the $\mathrm{V}$ category. The items with a lower criticality need and those whose non-availability of which the hospital can function nut can affect the quality of services, the non-availability can be managed for a small course of time. E category are those of whose unavailability may not interfere with the functioning. The VED status of each item was discussed with justification by the oncologists.

\section{$A B C-V E D$ matrix}

The matrix will help classify the drugs under vitality and consumption value. e.g. drugs of low consumption value and high criticality, drugs of high consumption value and high criticality and vice versa (Table 2). In this manner the management can focus on both high consumption value and criticality. ABC-VED matrix was formulated by cross-tabulating the $\mathrm{ABC}$ and VED analysis. From the resultant combination, three categories were classified (I, II and III). Category I was constituted by items belonging to $\mathrm{AV}, \mathrm{AE}, \mathrm{AD}, \mathrm{BV}$ and $\mathrm{CV}$ subcategories. The $\mathrm{BE}, \mathrm{CE}$ and $\mathrm{BD}$ subcategories constituted category II, and the remaining items in the CD subcategory constituted category III. In these subcategories, the first alphabet denotes its place in the ABC analysis, while the second alphabet stands for its place in the VED analysis.

\section{Inventory index}

This method can be used to calculate the stock turnover using opening stock, closing stock, average inventory, total and average per day consumption. The inventory index was calculated using the formula Inventory index is an indicator of stock turnover. First, the average value of inventory is Calculated based on the opening stock and closing stock. Then the average daily consumption is calculated. Finally, the inventory index is calculated by dividing the average value of inventory by the average consumption per day.

Annual Drug Expenditure $(\mathrm{ADE})=$ annual consumption of drug ${ }^{\star}$ unit cost of drug

2. Average value of inventory $=$ opening stock + closing stock $/ 2$

3. Average daily consumption $=$ Total consumption of month $/$ No. of days in month

4. Inventory Index = Average Value of Inventory/ Average value of consumption

\section{RESULTS}

Inventory Index ideally at the month end should be 0 or 1 , zero indicates a complete consumption and no stocking of inventory which is the most ideal as the stock turnover is fast and the stores are ready to receive the next consignment, inventory index of one indicates, the stock would suffice for a day's consumption, but this is difficult to achieve in tertiary care teaching hospital where there will be continuous and varying trends of patient flow. More the inventory index number lesser is the turnover of the inventory. Therefore, a single digit is considered for better inventory 
Table 1: ABC analysis

\begin{tabular}{cccc}
\hline & Category A & Category B & Category C \\
\hline Number of items & 4 & 4 & 161 \\
Percentage of items & $2.4 \%$ & $2.4 \%$ & $95.2 \%$ \\
$\begin{array}{c}\text { \% Value of annual } \\
\text { consumption }\end{array}$ & 69.32 & 20.27 & 10.41 \\
\hline
\end{tabular}

\section{Table 2: ABC - VED matrix}

\begin{tabular}{cccc}
\hline & $\mathbf{V}$ & $\mathbf{E}$ & $\mathbf{D}$ \\
\hline $\mathrm{A}$ & $\mathrm{AV}(0)$ & $\mathrm{AE}(4)$ & $\mathrm{AD}(0)$ \\
$\mathrm{B}$ & $\mathrm{BV}(0)$ & $\mathrm{BE}(4)$ & $\mathrm{BD}(0)$ \\
$\mathrm{C}$ & $\mathrm{CV}(1)$ & $\mathrm{CE}(152)$ & $\mathrm{CD}(8)$ \\
\hline
\end{tabular}

\begin{tabular}{cccccc}
\hline \multicolumn{2}{l}{ Table 3: Inventory index for category $\mathbf{1}$ drugs } \\
\hline Drug name & NOV & DEC & JAN & FEB & MARCH \\
\hline CAPECITABINE $500 \mathrm{mg}$ & 4.71 & 20.95 & 16.78 & 8.06 & 12.38 \\
TRASTUZUMAB $440 \mathrm{mg}$ & 15.00 & 7.75 & 9.30 & 21.75 & 10.33 \\
BEVACIZUMAB $400 \mathrm{mg} / 16 \mathrm{ml}$ & 0.00 & 0.00 & 0.00 & 5.80 & 7.75 \\
MELPHALAN $5 \mathrm{mg}$ & 20.83 & 9.01 & 16.15 & 8.06 & 48.44 \\
ARSENIC TRIOXDE $1 \mathrm{mg} / \mathrm{ml}$ & 14.42 & 0.00 & 9.54 & 31.52 & 25.95 \\
\hline
\end{tabular}

management. The values of our Inventory index are shown in Table 3. Data obtained from the pharmacy showed that only 29 drugs of the 169 drugs available in the oncology pharmacy were used in the past 6 months. The remaining 140 drugs were non-moving items. Of the 169 drugs included in the $\mathrm{ABC}$ analysis, 4 drugs were under classified under category A ( $70 \%$ of total cost-Rs.1,50,02,569). Category A formed $2.4 \%$ of the total drugs. The next $20 \%$ of total cost (Rs.43,82,251) was incurred by 4 drugs included in category B. Category also constituted $2.4 \%$ of the total list of drugs. The final $10 \%$ of the cost (Rs.22, 02,898) was due to 21 drugs included in Category C forming $95.2 \%$ of the total drugs. This is showed in Table 1.

\section{VED Analysis}

The list of 169 drugs available in the oncology pharmacy was subjected to VED analysis. One drug was classified as vital, 160 as essential and 8 as desirable. The result of VED analysis showed one drug as Vital (Rs. 2,27,500/-) drug with low cost expenditure. 8 drugs as Desirable and 160 drugs as Essential.

\section{Matrix Analysis}

Matrix analysis showed a total of 5 drugs in category 1 i.e. 4 drugs in high cost essential dugs and one in low cost vital drug show in Table 2. Category II had 156 drugs, i.e. 4 medium cost essential drugs and 152 low cost essential drugs. Cat III with 8 drugs that are low cost and desirable. This is shown in Table 2. Inventory Index of all the drugs is showed as per Table 3 .

\section{Inventory Index}

Inventory Index = Average Value of Inventory/month divided by Average value of consumption/ day. ure 03 shows inventory index for category 1 drugs

Inventory index can help store managers to know the stock availability at the closing month. i.e. how much stock availability and helps to minimize over stocking and understand the inventory turnover

Inventory index for cat I drugs:

Inventory index for 6 months of category 1 drugs are as follows

\section{DISCUSSION}

The present study showed that if only ABC method was used independently it could only help the administrators to focus on drugs that have a high annual drug expenditure is A category whereas the items that are critical may fall in category $\mathrm{B}$ or $\mathrm{C}$ hence the same may not receive the focus, management and supervisory control this finding is similarly seen in study conducted by devnani et al in northern India. ${ }^{14}$ The VED analysis may tend to bring the focus of administrators control more on vital and essential drugs, whereas the Annual drug expenditure for some of the vital or essential may be comparatively lower. Therefore, the matrix analysis will help to focus on all the range of criticality and their annual drug expenditure. The annual drug expenditure was (ADE) was Rs. 2,15,87,718.84/-. The ABC analysis showed around 169 oncology drugs are present in stock of pharmacy of oncology unit. Out of the available 169 drugs only 29 drugs were in consumption. From the 29 drugs, $13.79 \%$ (4) drugs fall under A cat (Rs. 1,50,02,569/-) which is high cost center i.e. $10 \%$ of drugs are attributing $70 \%$ of monetary value and another $13.79 \%$ (4) drugs lie under B cat (Rs. $43,82,251 /$-) i.e. $20 \%$ of drugs are attributing $20 \%$ of monetary value. The VED analysis resulted in from 169 drugs, only one drug i.e. ARESENIC TRIOXIDE $1 \mathrm{mg} / \mathrm{ml}$ is a Vital (Rs. 2,27,500/-) drug with low cost which lies in cat C in ABC analysis. Around 8 drugs were classified as Desirable (Rs. 53,028/-) and 160 drugs as Essential drugs (Rs. 21,307,190.28/-) and the rest $72.41 \%$ (21) drugs lie under C cat (Rs. 22,02,898/-) which are having low cost center i.e. $70 \%$ of drugs attributed to $10 \%$ of monetary value. The matrix analysis showed that of the five drugs in category 1 , total of four drugs comprised in the $\mathrm{AE}$ (high cost essential) and a single drug in the $\mathrm{CV}$ (low cost and vital) Similarly in cat 2, BE (medium cost essential) comprises of 4 drugs and CE (essential low cost) are 152 in quantity, 8 drugs of $\mathrm{CD}$ (desirable low cost) in cat 3.

The limitation of $\mathrm{ABC}$ analysis is that it is based only on monetary value and rate of consumption of the items. Sometimes, particularly in a hospital an item of low monetary value and consumption may be very vital or even lifesaving. Their importance cannot be overlooked simply because they do not appear in category A of inventory. Therefore, another parameter of the materials is their criticality. This could be in item of the therapeutic value of a drug or intrinsic value of the material in achieving the objectives of the hospital system. 8 Control exercise by managers should be such that efficient inventory system is in place for normal operating conditions and the hospital is also able to meet the emergency demand conditions $9.20 \%$ savings in medical stores of the hospital can be brought about by review and control measures for expensive drugs. ${ }^{10}$ If drug inventory is managed based solely on $\mathrm{ABC}$ analysis, supervision of only 19 drugs would have resulted in control over approximately $70 \%$ of the drug budget. The managers could save their time by ignoring to 
manage $70 \%$ of drugs which cost only $10 \%$ of the total expenditure on drugs. ${ }^{11}$

This study has shown how managers can also exercise control by calculating the inventory turnovers using the inventory index method. To improve the Turnover of Inventory, items should be standardized, rate contract and just in time (JIT) can be applied to as many items possible and good vendor relationship to ensure minimum external lead time. Close monitoring of non-moving items, negotiations and contracts with vendors to return the items if not under consumption to avoid unnecessary stocking and inventory costs and creating a culture of consciousness regarding material handling and usage among health care providers in the hospital.

This study gives information to managers to plan \& control by calculating the inventory turnovers using the inventory index method. To improve the Turnover of Inventory, items should be standardized, rate contract and just in time (JIT) was applied and good vendor relationship to ensure minimum external lead time. Close monitoring of non-moving items, negotiations and contracts with vendors to return the items if not under consumption to avoid unnecessary stocking and inventory costs and creating a culture of consciousness regarding material handling and usage among health care providers in the hospital.

\section{CONCLUSION}

Drugs present in Cat I requires stringent control from the top management and regular monthly maintenance figures for all category oncology drugs should be maintained and reported. Purchasing of oncology drugs can follow Just In Time (JIT) system to avoid over stock of drugs. Scheduling of chemo therapy treatment could be shared with the pharmacists so that they can arrange the drugs according to the schedule or help in purchase in case of JIT system. Good vendor relationship and negotiate terms and conditions on replacement of drugs in case of over stock. Implementation of automated system to measure the inventory index and other parameters of inventory control. Drug formulary should be reviewed periodically to remove nonmoving and drugs which are not in consumption for 3 months.

\section{REFERENCES}

1. Kumar S, Chakravarty A. ABC-VED analysis of expendable medical stores at a tertiary care hospital. Medical Journal Armed Forces India. 2015;71(1):24-7.

2. Singh S, Gupta A, Devnani M. ABC and VED Analysis of the Pharmacy Store of a Tertiary Care, Academic Institute of the Northern India to Identify the Categories of Drugs Needing Strict Management Control. Journal of Young Pharmacists. 2015;7(2):76-80.

3. Devnani M, Gupta AK, Nigah R. ABC and VED Analysis of the Pharmacy Store of a Tertiary Care Teaching, Research and Referral Healthcare Institute of India. Journal of Young Pharmacists. 2010;2(2):201-5.

4. Evaluating Hospital Pharmacy Inventory Management and Revenue Cycle Processes [Internet]. US: Association of health care internal auditors; 2017 [cited 26 January 2017]. Available from: https://www.ahia.org/assets/Uploads/ pdfUpload/WhitePapers/EvaluatingHospitalPharmacylnventoryManagement

5. Khurana S, Chhillar N, Gautam V. Inventory control techniques in medical stores of a tertiary care neuropsychiatry hospital in Delhi. 2017.

6. Gupta R, Gupta K, Jain B, Garg R. ABC and VED Analysis in Medical Stores Inventory Control. Medical Journal Armed Forces India. 2007;63(4):325-7.

7. Mahatme M, Hiware S, Shinde A, Salve A, Dakhale G. Medical Store Management: An Integrated Economic Analysis of a Tertiary Care Hospital in Central India. Journal of Young Pharmacists. 2012;4(2):114-8.

8. Hospital Stores Management an Integrated Approach. 1st ed. jaypee; 2017.

9. [Internet]. 2017 [cited 26 September 2017]. Study on MMF and Inventory Index on Inventory Management Practices at a Medical College Teaching Hospital; Available from: http://www.iosrjournals.org/iosr-jbm/papers/Nol17-issue4/Nersion-2/ G017426065.pdf

10. Duclos L. Hospital Inventory Management for Emergency Demand. International Journal of Purchasing and Materials Management. 1993;29(3):29-38.

11. Pillans P, Conry I, Gie B. Drug Cost Containment at a Large Teaching Hospital. PharmacoEconomics. 1992;1(5):377-82.

Article History: Submission Date : 03-05-2017 ; Revised Date : 01-06-2017; Acceptance Date : 29-09-2017.

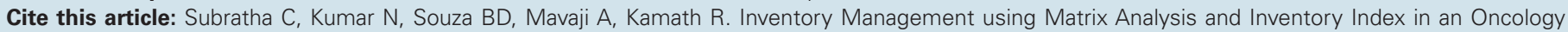
Pharmacy of a Tertiary Care Teaching Hospital. J Young Pharm. 2018;10(1):78-81. 